\title{
Imaging Identification of Rapidly Progressing Autosomal Dominant Polycystic Kidney Disease: Simple Eligibility Criterion for Tolvaptan
}

\author{
$E_{i j i ~ H i g a s h i h a r a}{ }^{a, b}$ Shigeo Horie ${ }^{c}$ Satoru Muto ${ }^{c}$ Haruna Kawano $^{c}$ \\ Mitsuhiro Tambo $^{b}$ Tsuyoshi Yamaguchi ${ }^{b}$ Satoru Taguchi ${ }^{b}$ Shinya Kaname ${ }^{d}$ \\ Kenich Yokoyama ${ }^{\mathrm{e}}$ Tatsuya Yoshioka ${ }^{\mathrm{e}}$ Toshihito Furukawa $^{\mathrm{f}}$ \\ Hiroshi Fukuharac \\ aDepartment of Hereditary Kidney Disease Research, Kyorin University School of Medicine, Tokyo, Japan; \\ ${ }^{b}$ Department of Urology, Kyorin University School of Medicine, Tokyo, Japan; 'Department of Urology, Juntendo

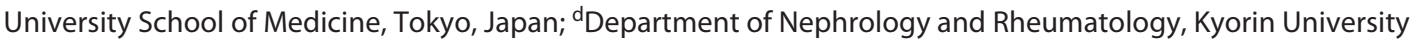 \\ School of Medicine, Tokyo, Japan; 'Department of Radiology, Kyorin University School of Medicine, Tokyo, Japan; \\ fBiostatistical Research Co., Ltd., Tokyo, Japan
}

\section{Keywords}

Autosomal dominant polycystic kidney disease $\cdot$ Total kidney volume $\cdot$ Height-adjusted TKV $\cdot$ Estimated HtTKV growth rate End-stage renal disease - Tolvaptan .

Guidelines

\begin{abstract}
Background: Tolvaptan was approved for the treatment of autosomal dominant polycystic kidney disease (ADPKD). However, the official indication of "rapidly progressive disease" is described differently in the clinical guidelines. We aim to define "rapidly progressive disease" by risk of ESRD, which is evaluated using estimated height-adjusted total kidney volume (HtTKV) growth rate. Methods: The risk of ESRD was retrospectively analyzed in 617 initially non-ESRD adults with ADPKD and observed with standard of care between 2007 and 2018. The estimated annual growth rate of the HtTKV, termed as eHTKV-a (\%/year), is derived from the following equation: [HtTKV at age $t]=\mathrm{K}(1+\mathrm{eHTKV}-\mathrm{a} / 100)^{t}$,
\end{abstract}

where $K=150 \mathrm{~mL} / \mathrm{m}$ is used in Mayo Imaging Classification and $K=130 \mathrm{~mL} / \mathrm{m}$ is proposed for individually stable eHTKV-a value from baseline. The accuracy of eHTKV-a to predict ESRD for censored ages was analyzed using time-dependent receiver-operating characteristic curves (ROC). The cutoff point of initially measured eHTKV-a to predict ESRD was assessed using Kaplan-Meier and Cox's proportional hazards models. Performance characteristics of the cutoff point for censored ages were calculated using time-dependent ROC and validated by the bootstrap method. Results: The area under the time-dependent ROC of eHTKV-a to predict ESRD at age 65 was $0.89 \pm 0.04(K=130)$. The mean renal survival was less than 70 years at eHTKV-a $\geq 4.0 \%$ /year $(K=130)$. Mean renal survival was approximately 12 years shorter, and hazard ratio of ESRD was more than 5-time higher at this cutoff point than at lower point. Time-dependent sensitivity for age 65 and cutoff point of $4.0 \% /$ year $(K=130)$ was $93.4 \pm$ $0.3 \%$. Between cutoff points $\geq 4.0 \% /$ year $(K=130)$ and $\geq 3.5 \% /$ year $(K=150)$, there was no significant difference in performance characteristics and accuracy to predict ESRD. Conclu- karger@karger.com www.karger.com/ajn

Karger $\stackrel{\text { ' }}{5}$

GOPEN ACCESS
(C) 2020 The Author(s)

Published by S. Karger AG, Basel

This is an Open Access article licensed under the Creative Commons Attribution-NonCommercial-4.0 International License (CC BY-NC) (http://www.karger.com/Services/OpenAccessLicense), applicable to the online version of the article only. Usage and distribution for commercial purposes requires written permission.
Eiji Higashihara

Department of Hereditary Kidney Disease Research, Kyorin University School of Medicine 6-20-2 Shinkawa, Mitaka

Tokyo 181-8611 (Japan)

ehigashi@ks.kyorin-u.ac.jp 
sion: eHTKV-a well predicts ESRD. Initially, measured eHTKV- $a \geq 4.0 \% /$ year $(K=130)$ defines high-risk ESRD. Without additional conditions, a single eHTKV-a cutoff point identifies subjects that are most likely to benefit from tolvap$\tan$.

(C) 2020 The Author(s)

Published by S. Karger AG, Basel

\section{Introduction}

Autosomal dominant polycystic kidney disease (ADPKD) is an inherited kidney disease and fourth leading cause of ESRD [1,2]. Approximately $70 \%$ of patients with ADPKD progress to ESRD at around the age of 60-70 years $[3,4]$. The wide variations in ages of reaching ESRD are accounted for by different rates of total kidney volume (TKV) enlargement $[1,5-7]$. The Tolvaptan Efficacy and Safety in Management of ADPKD and its Outcomes (TEMPO) 3:4 trial reported beneficial effects of tolvaptan, a selective vasopressin V2-receptor antagonist, to slow the growth rate in TKV and the decline rate in kidney function in patients with ADPKD [8].

In acknowledgment of the TEMPO 3:4 results, the Ministry of Health, Labor, and Welfare in Japan approved tolvaptan in March 2014 for the treatment of patients with ADPKD, TKV of $750 \mathrm{~mL}$ or more, and TKV growth rate of 5\%/year or more $[9,10]$. In February 2015, Health Canada approved tolvaptan for patients with ADPKD with no initial specification [11]. The European Medical Agencies followed in May 2015, approving tolvaptan for the treatment of ADPKD patients with evidence of rapid disease progression [12]. After confirming tolvaptan benefits in later-stage ADPKD [13], the US Food and Drug Administration approved the use of tolvaptan as a treatment in adults at risk of rapidly progressing ADPKD in April 2018 [14].

"Rapidly progressing disease" was proposed by the European Medical Agencies and Food and Drug Administration as an eligibility criterion for tolvaptan treatment for ADPKD. However, practical guides of EU and US defined "rapidly progressing disease" differently $[15,16]$. The European Renal Association-EDTA Working Groups on Inherited Kidney Disorders and European Renal Best Practice (ERA-EDTA) defined "rapidly progressing disease" basically by the estimated glomerular filtration rate (eGFR) indexed for age. As the Consortium of Radiologic Imaging Studies of Polycystic Kidney Disease [5] revealed that eGFR cannot predict ESRD for several reasons, a US group used the Mayo Imaging Classification (Mayo Class) [6] to identify "rapidly progressing disease" [16].
The Mayo Class was proposed as a simple model to predict renal prognosis in adults with typical ADPKD. The Mayo Class is distinguished using the estimated height-adjusted TKV (HtTKV) growth rate (\%/year), which was abbreviated as eHTKV- $\alpha$ [17]. eHTKV- $\alpha$ is calculated using the following equation: [HtTKV at age $t]=K \times(1+\text { eHTKV- } \alpha / 100)^{t}$, where $K=150 \mathrm{~mL} / \mathrm{m}$ is used [6].

In Mayo Class, the eHTKV- $\alpha$ is applied to typical-type ADPKD adults of arbitrary age. However, the individual eHTKV- $\alpha$ changed and more than 1 out of 5 of patients later moved to another prognostic category [6]. The constancy of individual eHTKV- $\alpha$ over years is preferable for prognostic application of eHTKV-a. In our previous study, $K=130 \mathrm{~mL} / \mathrm{m}$ yielded a more stable individual eHTKV- $\alpha$ from baseline [17].

EU and US guidelines regard Mayo Class $1 \mathrm{C}$ to $1 \mathrm{E}$ subclasses as "rapid disease progression" $[15,16]$. However, additional risk factors are required for tolvaptan treatment in patients classified as 1C by Canadian guidelines [18]. Five Mayo Classes (eHTKV- $\alpha$ of -1.5, 1.5-3.0, 3.0$4.5,4.5-6.0$, and 6.0-\%/year) are categorized as slow, intermediate, and rapid progression [6]. Hence, class 1C (3.0-4.5\%/year) is originally classified into the intermediate progression category.

As eGFR decline is individually variable and nonlinear in a substantial fraction [19], the risk of ESRD is adopted as a more reliable outcome measure of rapidly progressive disease. We aimed to specify an eHTKV- $\alpha$ cutoff point to more precisely define subjects at high-risk of ESRD.

\section{Patients and Methods}

Study Design

The present study aimed to identify "rapidly progressive ADPKD" by risk of ESRD using eHTKV- $\alpha$ data from 2 Japanese ADPKD cohorts. Two cutoff points of eHTKV-a were calculated using $K=130 \mathrm{~mL} / \mathrm{m}$ and $K=150 \mathrm{~mL} / \mathrm{m}$ for comparison of accuracy and performance. The accuracy of eHTKV-a for predicting ESRD was examined by the area under the time-dependent receiver-operating characteristic curve (ROC). Preliminary cutoff points of eHTKV - $\alpha$ to predict the eGFR declining to $15 \mathrm{~mL} / \mathrm{min} / 1.73 \mathrm{~m}^{2}$ at censored ages 60,65 , and 70 were calculated. Censored ages were selected because the incidence of renal replacement therapy (RRT) is high from age 50 to 75 in 2006-2010 ERA-EDTA data [4].

The mean renal survival and hazard ratio of ESRD were compared among several subgroups divided variably around preliminary cutoff points of eHTKV-a to identify those to predict highrisk ESRD. Then, performance characteristics of eHTKV- $\alpha$ as a predictor of ESRD for the cutoff points and for different censored ages were estimated using time-dependent ROC analysis and vali- 


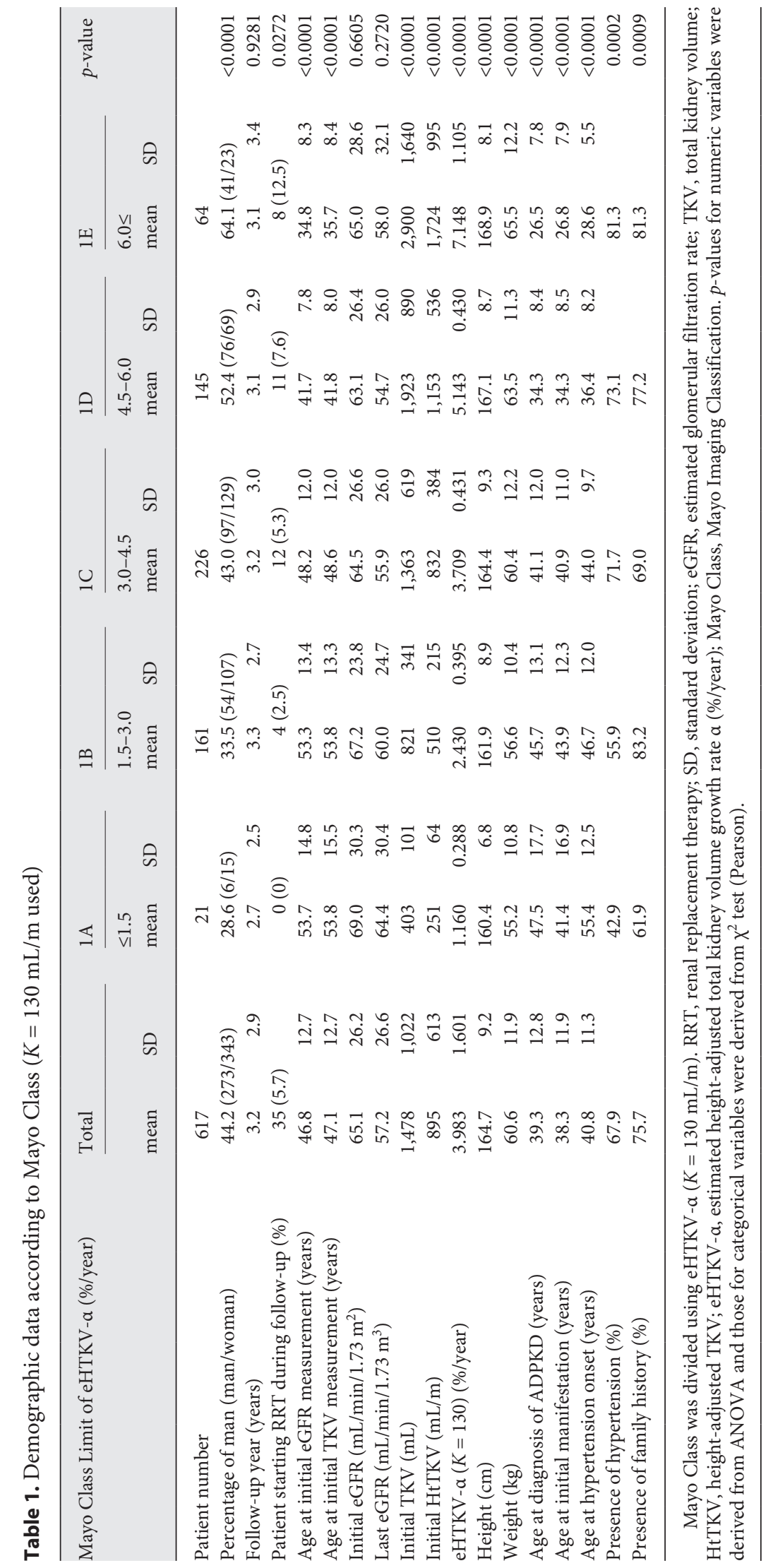


Table 2. Validation of the area under the time-dependent ROC curve

\begin{tabular}{|c|c|c|c|c|}
\hline \multirow[t]{2}{*}{ Factor } & \multirow{2}{*}{$\begin{array}{l}\text { Censored } \\
\text { age }\end{array}$} & \multirow{2}{*}{$\begin{array}{l}\text { AUC of entire } \\
\text { data set }\end{array}$} & \multicolumn{2}{|c|}{ Validated AUC } \\
\hline & & & mean $\pm \mathrm{SE}$ & $95 \% \mathrm{CI}$ \\
\hline \multirow[t]{3}{*}{ eHTKV-a $(K=130)$} & 60 & 0.8187 & $0.882 \pm 0.049$ & $0.786-0.978$ \\
\hline & 65 & 0.8233 & $0.892 \pm 0.038$ & $0.817-0.966$ \\
\hline & 70 & 0.7982 & $0.876 \pm 0.045$ & $0.788-0.965$ \\
\hline \multirow[t]{3}{*}{ eHTKV-a $(K=150)$} & 60 & 0.8256 & $0.876 \pm 0.051$ & $0.776-0.975$ \\
\hline & 65 & 0.8271 & $0.884 \pm 0.040$ & $0.806-0.963$ \\
\hline & 70 & 0.8017 & $0.866 \pm 0.047$ & $0.775-0.958$ \\
\hline \multirow[t]{2}{*}{ Factor } & \multirow{2}{*}{$\begin{array}{l}\text { Censored } \\
\text { age }\end{array}$} & \multirow{2}{*}{$\begin{array}{l}\text { Difference } \\
\text { in AUC }\end{array}$} & \multicolumn{2}{|c|}{ Difference in validated AUC } \\
\hline & & & mean $\pm \mathrm{SE}$ & $95 \% \mathrm{CI}$ \\
\hline \multirow[t]{3}{*}{ eHTKV- $\alpha(K=130)-\mathrm{eHTKV}-\alpha(K=150)$} & 60 & -0.0069 & $0.006 \pm 0.003$ & $0.001-0.011$ \\
\hline & 65 & -0.0039 & $0.008 \pm 0.004$ & $0.001-0.015$ \\
\hline & 70 & -0.0035 & $0.010 \pm 0.004$ & $0.002-0.018$ \\
\hline
\end{tabular}

The area under the ROC curve was calculated using time-dependent ROC analysis and was validated using the Uno method [23]. eHTKV-a, estimated height-adjusted total kidney volume growth rate a (\%/year); SE, standard error; CI, confidence interval; AUC, area under the ROC curve; ROC, receiver operating characteristic.

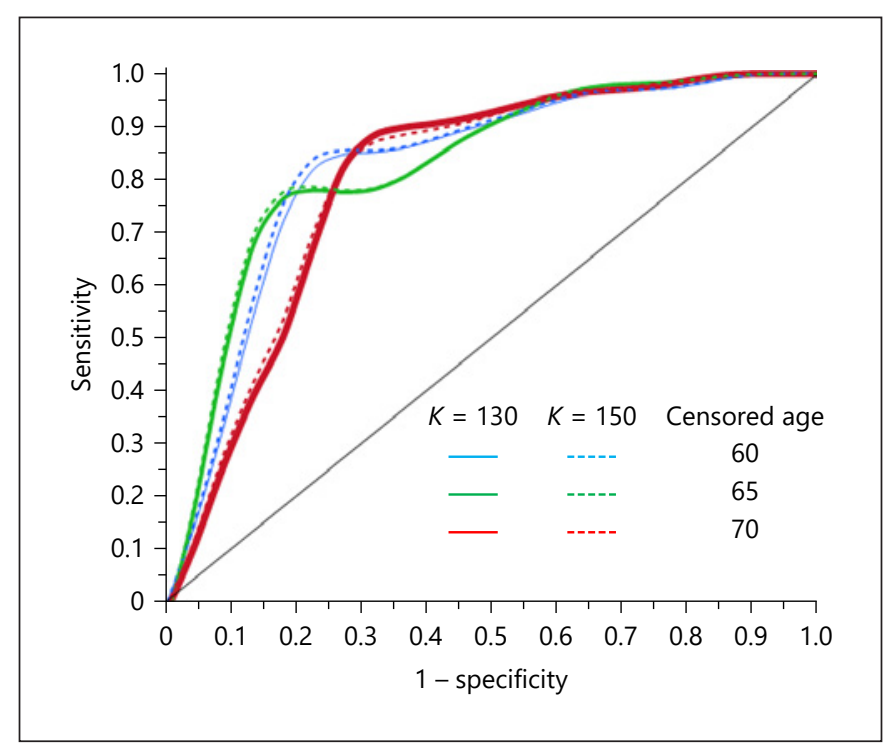

Fig. 1. eHTKV-a well predicts ESRD. The AUC of eHTKV- $\alpha$ was estimated using the entire data set (Table 2). AUC, area under the ROC curve; eHTKV- $\alpha$, estimated height-adjusted total kidney volume growth rate $a$ (\%/year). dated using the bootstrap method. The Institutional Review Boards of Kyorin University (1046-04) and Juntendo University (19-148) approved this study and all patients provided informed consent.

\section{Study Participants}

A total of 617 standard-of-care patients with typical ADPKD and baseline age above 19 who visited Kyorin University Hospital (388 patients) and Juntendo University Hospital (229 patients) between April 2007 and December 2018 were analyzed. Subjects under the age of 20 were excluded because the equation for eHTKV- $a$ is derived from the analysis of adult data $[6,17]$. Patients who started RRT prior to the initial visit or who received invasive therapy or tolvaptan to reduce the renal volume at any time were excluded from the analysis.

Measurement of TKV and eGFR and the Definition of ESRD

TKV was measured using stereology by MRI [20]. eGFR was calculated using serum $\mathrm{Cr}$ according to the Isotope Dilution Mass Spectrometry and Modification of Diet in Renal Disease (IDMSMDRD) with the Japanese coefficient [21]. ESRD was defined by starting RRT (kidney transplantation or dialysis) during the observation period, and the case was truncated in renal survival analyses.

\section{$e H T K V-\alpha$ Calculations}

Using the equation [HtTKV at age $t]=K(1+\mathrm{eHTKV}-\alpha / 100)^{t}$, eHTKV- $\alpha$ was calculated with age measured to 2 digits after the decimal point. $K=130 \mathrm{~mL} / \mathrm{m}$ and $K=150 \mathrm{~mL} / \mathrm{m}$ were used [17].

\section{Estimation of Preliminary eHTKV- $\alpha$ Cutoff Values to Predict} CKD Stage 5

The eGFR at age 60,65 , and 70 was estimated using individual eGFR regression lines. Preliminary cutoff values of eHTKV-a to 
Table 3. Comparison of renal survival among subgroups divided by eHTKV-a around preliminary cutoff values

\begin{tabular}{|c|c|c|c|c|c|c|}
\hline \multirow{2}{*}{$\begin{array}{l}\text { Comparison subgroups } \\
\text { divided by initial } \\
\text { eHTKV-a (\%/year) }\end{array}$} & \multicolumn{2}{|c|}{ Truncated/censored number } & \multicolumn{4}{|c|}{ Renal survival (years) } \\
\hline & $K=130$ & $K=150$ & $\begin{array}{l}\text { mean } \pm \mathrm{SE} \\
K=130\end{array}$ & $p$-value & $\begin{array}{l}\text { mean } \pm \mathrm{SE} \\
K=150\end{array}$ & $p$-value \\
\hline \multicolumn{7}{|c|}{ a Four comparison subgroups } \\
\hline$\leq 2.0$ & $0 / 51$ & $0 / 81$ & - & $<0.0001$ & - & $<0.0001$ \\
\hline $2.0<\cdots \leq 3.0$ & $4 / 127$ & $6 / 148$ & $71.9 \pm 0.3$ & & $71.5 \pm 0.4$ & \\
\hline $3.0<\cdots \leq 4.0$ & $8 / 149$ & $8 / 142$ & $74.6 \pm 0.9$ & & $74.3 \pm 1.0$ & \\
\hline $4.0<$ & $23 / 255$ & $21 / 211$ & $63.5 \pm 1.0$ & & $62.1 \pm 1.3$ & \\
\hline$\leq 2.5$ & $2 / 104$ & $3 / 146$ & $67.8 \pm 0.3$ & $<0.0001$ & $72.1 \pm 0.3$ & $<0.0001$ \\
\hline $2.5<\cdots \leq 3.5$ & $6 / 154$ & $7 / 158$ & $71.5 \pm 0.5$ & & $75.3 \pm 0.7$ & \\
\hline $3.5<\cdots \leq 4.5$ & $8 / 134$ & $6 / 136$ & $73.2 \pm 1.3$ & & $67.2 \pm 0.7$ & \\
\hline $4.5<$ & $19 / 190$ & $19 / 142$ & $57.7 \pm 0.7$ & & $56.7 \pm 0.8$ & \\
\hline$\leq 3.0$ & $4 / 178$ & $6 / 229$ & $72.1 \pm 0.2$ & $<0.0001$ & $71.8 \pm 0.3$ & $<0.0001$ \\
\hline $3.0<\cdots \leq 4.0$ & $8 / 149$ & $8 / 142$ & $74.6 \pm 0.9$ & & $74.3 \pm 1.0$ & \\
\hline $4.0<\cdots \leq 5.0$ & $6 / 128$ & $6 / 115$ & $66.8 \pm 0.9$ & & $65.0 \pm 1.5$ & \\
\hline $5.0<$ & $17 / 127$ & $15 / 96$ & $56.2 \pm 1.0$ & & $55.6 \pm 1.2$ & \\
\hline$\leq 3.5$ & $8 / 258$ & $10 / 304$ & $71.7 \pm 0.3$ & $<0.0001$ & $75.7 \pm 0.4$ & $<0.0001$ \\
\hline $3.5<\cdots \leq 4.5$ & $8 / 134$ & $6 / 136$ & $73.2 \pm 1.3$ & & $67.2 \pm 0.7$ & \\
\hline $4.5<\cdots \leq 5.5$ & $6 / 100$ & $10 / 82$ & $55.9 \pm 0.4$ & & $58.0 \pm 0.9$ & \\
\hline $5.5<$ & $13 / 90$ & $9 / 60$ & $55.2 \pm 1.4$ & & $50.7 \pm 0.8$ & \\
\hline \multicolumn{7}{|c|}{ b Two comparison subgroups } \\
\hline$\leq 3.0$ & $4 / 178$ & $6 / 229$ & $72.1 \pm 0.2$ & $<0.0001$ & $71.8 \pm 0.3$ & $<0.0001$ \\
\hline $3.0<$ & $31 / 404$ & $29 / 353$ & $71.4 \pm 0.9$ & & $70.9 \pm 1.0$ & \\
\hline$\leq 3.5$ & $8 / 258$ & $10 / 304$ & $71.7 \pm 0.3$ & $<0.0001$ & $75.7 \pm 0.4$ & $<0.0001$ \\
\hline $3.5<$ & $27 / 324$ & $25 / 278$ & $69.6 \pm 1.3$ & & $64.0 \pm 0.9$ & \\
\hline$\leq 4.0$ & $12 / 327$ & $14 / 371$ & $75.5 \pm 0.4$ & $<0.0001$ & $75.4 \pm 0.4$ & $<0.0001$ \\
\hline $4.0<$ & $23 / 255$ & $21 / 211$ & $63.5 \pm 1.0$ & & $62.1 \pm 1.3$ & \\
\hline$\leq 4.5$ & $16 / 392$ & $16 / 440$ & $75.2 \pm 0.4$ & $<0.0001$ & $75.2 \pm 0.4$ & $<0.0001$ \\
\hline $4.5<$ & $19 / 190$ & $19 / 142$ & $57.7 \pm 0.7$ & & $56.7 \pm 0.8$ & \\
\hline Total & $35 / 582$ & & $73.6 \pm 0.5$ & & $73.6 \pm 0.5$ & \\
\hline
\end{tabular}

Results were derived from Kaplan-Meier estimates using eHTKV- $\alpha$, calculated using $K=130 \mathrm{~mL} / \mathrm{m}$ and $K=$ $150 \mathrm{~mL} / \mathrm{m}$. Comparison subgroups were divided around an eHTKV- $\alpha$, of $4 \% / y e a r$, which was a preliminary cutoff value of the initial eHTKV-a, to predict eGFR, decline to $15 \mathrm{~mL} / \mathrm{min} / 1.73 \mathrm{~m}^{2}$ at age 60 to 70 (online suppl. Table 2). Cutoff points printed in italic and bold were selected based on: (1) the mean renal survival $\leq 70$ years at an eHTKV- $\alpha \geq$ the cutoff point, (2) difference in mean renal survival $\geq 5$ years between 2 subgroups divided by cutoff points, and (3) smaller cutoff points of eHTKV-a, in each K-value. eHTKV-a, estimated height-adjusted total kidney volume growth rate (\%/year); SE, standard error. $p$-values were derived from the log-rank and Wilcoxon tests.

predict CKD stage $5\left(\mathrm{eGFR}<15 \mathrm{~mL} / \mathrm{min} / 1.73 \mathrm{~m}^{2}\right)$ at censored ages were estimated using ROC analysis.

Evaluation of Accuracy of eHTKV- $\alpha$ to Predict ESRD

The accuracy of eHTKV- $\alpha$ to predict ESRD for censored ages was evaluated using the area under time-dependent ROC (AUC) [22] and validated using Uno's Concordance Statistic [23].

Estimation and Validation of eHTKV- $\alpha$ Cutoff Points to Predict High-Risk ESRD

The mean renal survival was compared according to the subgroups divided by a $0.5 \% /$ year interval of eHTKV- $\alpha$ around pre- liminary cutoff values. As a standard definition of rapidly progressive disease was absent, the cutoff point for high-risk ESRD was defined as follows: (1) mean renal survival was less than 70 years in the subgroup with an eHTKV- $\alpha \geq$ the cutoff point (high-risk of ESRD). (2) The mean renal survival in the subgroup with an eHTKV- $\alpha \geq$ the cutoff point was shorter than that of subgroups with an eHTKV- $\alpha<$ cutoff point by 5 years. (3) A lower eHTKV- $\alpha$ was selected as the cutoff point among cutoff values that satisfied criteria (1) and (2). (4) The hazard ratio of ESRD of the high-risk subgroup compared with the subgroups with the next lower eHTKV- $\alpha$ value was confirmed to be significantly higher afterward. The performance characteristics of the cutoff point of 
eHTKV- $\alpha$ to predict ESRD for censored ages were validated using the bootstrap method.

\section{Statistical Analysis}

Normally distributed variables are expressed as the mean \pm standard error or standard deviation. Differences between groups were tested using the $\chi^{2}$ test for categorical variables and a general linear model with covariates as factors for continuous variables.

Regression lines of eGFR against age of the subgroups divided by the initial eHTKV-a were calculated using a mixed-effect model with age, groups, and sex as fixed effects, and with age $\times$ groups and age $\times$ sex as interaction effects. The mean renal survival was calculated using Kaplan-Meier analysis and multivariate hazard ratios adjusted by sex were calculated using Cox's proportional hazards model. AUC was estimated using time-dependent ROC analysis and was validated using Uno's method [23].

Performance characteristics of eHTKV- $a$ to predict ESRD for cutoff points and for censored ages were evaluated using timedependent ROC [22], and the sensitivity and specificity of the ESRD-predicting model were evaluated using the bootstrap method. Analyses were performed using SAS 9.4 and JMP Pro 14.3.0. A two-sided $p<0.05$ was considered significant.

\section{Results}

\section{Clinical Characteristics of Participants}

The demographic characteristics of the participants are summarized according to Mayo Class (Table 1). During mean ( \pm standard deviation) $3.2( \pm 2.6)$ years followup, eGFR decreased $8.0 \pm 11.9 \mathrm{~mL} / \mathrm{min} / 1.73 \mathrm{~m}^{2}$. Totally 35 patients started RRT during follow-up. The disease was more severe with a higher initial eHTKV-a. Earlier diagnosis, earlier onset of disease manifestations, and higher proportion of starting RRT were noted in higher eHTKV-a subgroups.

\section{Accuracy of eHTKV- $\alpha$ to Predict ESRD}

The validated AUC of eHTKV - $\alpha$ to predict ESRD for censored age 65 was $0.89 \pm 0.04$ and $0.88 \pm 0.04$ for $K=$ 130 and $K=150$, respectively (Table 2). The high AUC shows that eHTKV- $\alpha$ proposes good prediction of ESRD.

The validated AUC of $K=130 \mathrm{~mL} / \mathrm{m}$ was slightly higher than that of $K=150 \mathrm{~mL} / \mathrm{m}$ for censored ages (Table 2). However, when calculated using entire data set, the AUC was slightly higher for $K=150 \mathrm{~mL} / \mathrm{m}$ than for $K=130$ $\mathrm{mL} / \mathrm{m}$ for censored ages (Table 2; Fig. 1 ) and for all age ( $p=0.0693$, see online suppl. Fig. 1; for all online suppl. material, see www.karger.com/doi/10.1159/000511797). Take these results into together, there is no significant difference in AUC between $K=130 \mathrm{~mL} / \mathrm{m}$ and $K=150$ $\mathrm{mL} / \mathrm{m}$.
Cutoff Points of eHTKV- $\alpha$ to Predict CKD Stage 5

The eGFR slope became steeper from Mayo Class 1A to $1 \mathrm{E}$. The pattern of decline in eGFR was not noticeably different between $K=130 \mathrm{~mL} / \mathrm{m}$ and $K=150 \mathrm{~mL} / \mathrm{m}$ (online suppl. Table 1; Fig. 2).

Cutoff values of initial eHTKV-a to predict CKD stage 5 at censored ages were estimated from 4.0 to $4.3 \%$ /year (online suppl. Table 2). As the accuracy of the initial eHTKV- $\alpha$ to predict CKD stage 5 was weak $(A U C=0.63)$, these cutoff values were used as preliminary.

\section{Cutoff Points of eHTKV- $\alpha$ to Predict High-Risk ESRD}

Renal survival probability curves are illustrated for 4 subgroups divided by the initial eHTKV- $\alpha(\leq 3.0,3.0-4.0$, 4.0-5.0, and 5.0 $\leq$ ) (online suppl. Fig. 3). Renal survival probability was less than $50 \%$ for subjects with eHTKV- $\alpha$ $>4.0 \% /$ year at age $60-70$. This result is in accordance with the censored ages used in this study.

The mean renal survival for all subjects was 73.6 years (Table 3 ), and 70 years was selected as a limit of the mean renal survival for subgroups with a high-risk of ESRD. In the subgroups with a lower limit of eHTKV- $\alpha$ of $4.0 \%$ / year $(K=130 \mathrm{~mL} / \mathrm{m})$ or $3.5 \% /$ year $(K=150 \mathrm{~mL} / \mathrm{m})$, the mean renal survival was less than 70 years (Table $3 \mathrm{~A})$. In the subgroups with an eHTKV- $\alpha$ in these ranges, the mean renal survival was approximately 12 years shorter than that of the other subjects (Table 3B). Among cutoff points shown in Table 3, an eHTKV- $\alpha$ of $4 \% /$ year $(K=$ $130 \mathrm{~mL} / \mathrm{m})$ or $3.5 \% /$ year $(K=150 \mathrm{~mL} / \mathrm{m})$ was the lowest value and considered as a cutoff point for high-risk ESRD.

Multivariate hazard ratios of ESRD adjusted by the sex of the subgroup with high-risk ESRD versus the next lower eHTKV- $\alpha$ subgroup, or versus the other subjects were 7 to 8 - or 10 to 11 -times higher, respectively $(p<0.0001)$ (online suppl. Table 3).

\section{Performance Characteristics of eHTKV- $\alpha$ to Predict ESRD}

Time-dependent sensitivities to predict ESRD were between 97 and $98 \%$ for cutoff points of 4\%/year $(K=130$ $\mathrm{mL} / \mathrm{m})$ or $3.5 \% /$ year $(K=150 \mathrm{~mL} / \mathrm{m})$ and for censored ages 60,65 , and 70 . Time-dependent specificities were between 24 and $31 \%$ for the same cutoff points and ages (Table 4). For censored age 65, the mean sensitivity ( \pm SE) was $93.4 \pm 0.3 \%$ (95\% CI, 92.8-94.0\%) and $94.6 \pm 0.3 \%$ (95\% CI, $94.1-95.1 \%)$ for cutoff points $4.0 \% /$ year $(K=$ $130 \mathrm{~mL} / \mathrm{m})$ and $3.5 \% /$ year $(K=150 \mathrm{~mL} / \mathrm{m})$, respectively (Table 5). The subject numbers included in the high-risk subgroup were $278(45.1 \%$, for $K=130 \mathrm{~mL} / \mathrm{m})$ and 303 $(49.1 \%$, for $K=150 \mathrm{~mL} / \mathrm{m})$ for these cutoff points. 
Table 4. Performance characteristics of eHTKV- $\alpha$ to predict ESRD for censored ages and for the cutoff points

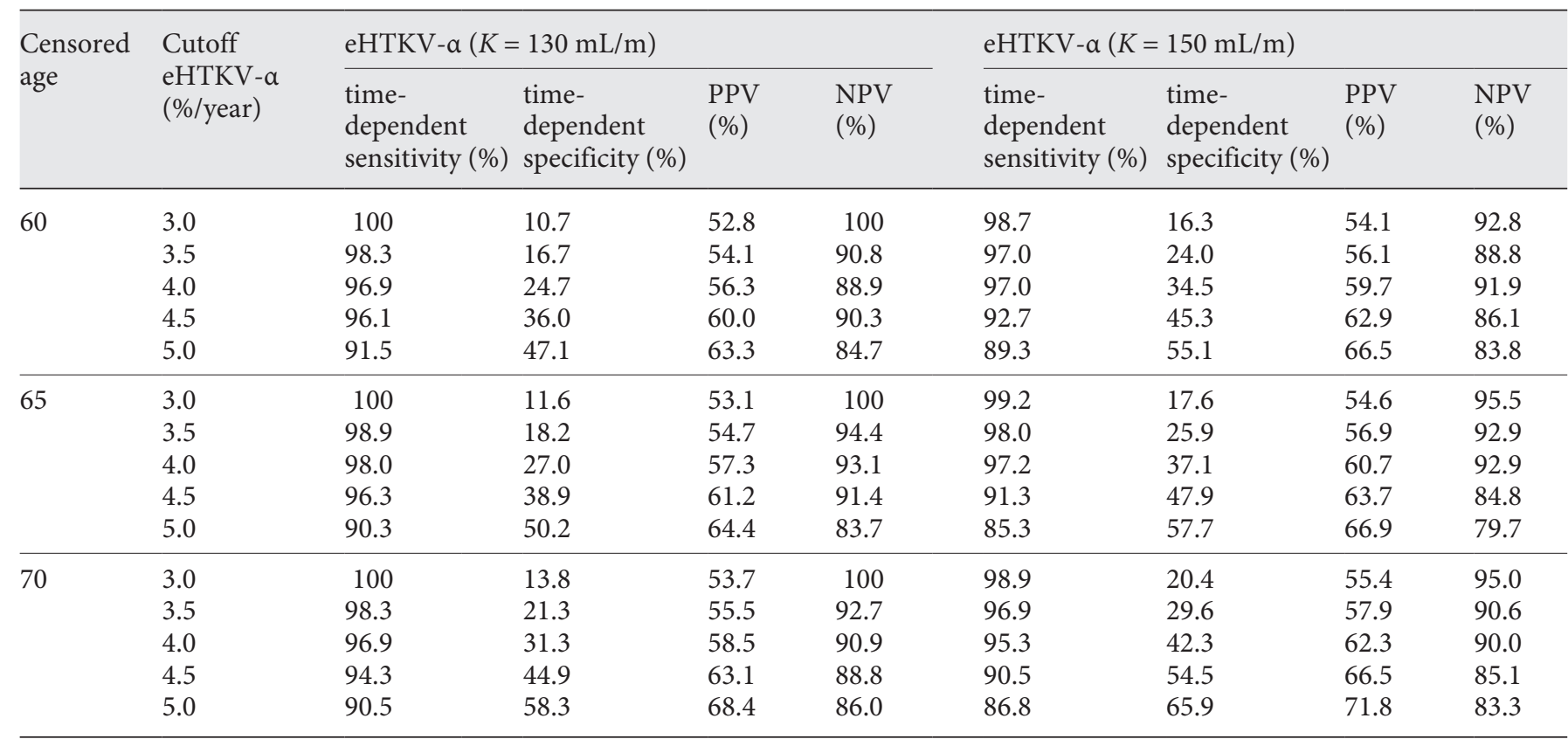

Performance characteristics were calculated using time-dependent ROC curve analysis. eHTKV- $\alpha$, estimated height-adjusted total kidney volume growth rate a (\%/year); PPV, positive predictive value; NPV, negative predictive value; ROC, receiver-operating characteristic.

Table 5. Validation of performance characteristics of the selected cutoff points to predict ESRD for different censored ages

\begin{tabular}{|c|c|c|c|c|c|c|}
\hline \multirow{2}{*}{$\begin{array}{l}\text { Censored } \\
\text { age }\end{array}$} & \multirow{2}{*}{$\begin{array}{l}\text { Cutoff } \\
\text { eHTKV-a }\end{array}$} & \multirow{2}{*}{$\begin{array}{l}\text { Equation } \\
\text { constant } K\end{array}$} & \multicolumn{2}{|c|}{ Time-dependent sensitivity (\%) } & \multicolumn{2}{|c|}{ Time-dependent specificity (\%) } \\
\hline & & & mean $\pm \mathrm{SE}$ & $95 \% \mathrm{CI}$ & mean $\pm \mathrm{SE}$ & $95 \% \mathrm{CI}$ \\
\hline \multirow[t]{2}{*}{60} & 4.0 & 130 & $92.54 \pm 0.28$ & 91.99-93.09 & $41.55 \pm 0.49$ & $40.59-42.50$ \\
\hline & 3.5 & 150 & $93.51 \pm 0.25$ & $93.02-94.01$ & $36.56 \pm 0.43$ & $35.72-37.39$ \\
\hline \multirow[t]{2}{*}{65} & 4.0 & 130 & $93.40 \pm 0.30$ & $92.81-93.99$ & $46.71 \pm 0.55$ & $45.63-47.79$ \\
\hline & 3.5 & 150 & $94.64 \pm 0.25$ & $94.14-95.14$ & $40.69 \pm 0.49$ & $39.73-41.65$ \\
\hline \multirow[t]{2}{*}{70} & 4.0 & 130 & $88.18 \pm 0.40$ & $87.40-88.96$ & $49.32 \pm 0.63$ & $48.07-50.56$ \\
\hline & 3.5 & 150 & $90.23 \pm 0.36$ & $89.53-90.93$ & $42.89 \pm 0.57$ & $41.78-44.00$ \\
\hline
\end{tabular}

The validity of sensitivity and specificity of the ESRD-predicting model using age was evaluated using the bootstrap method with 1,000 simulations. Thirty-five and 582 cases were randomly sampled with replacement from 35 ESRD and 582 non-ESRD cases, respectively. eHTKV- $\alpha$, estimated height-adjusted total kidney volume growth rate a (\%/year); SE, standard error; CI, confidence interval.

\section{Discussion}

Genic and allelic heterogeneity [3], possible modifying genetic factors [24], and environmental factors, such as treatment of hypertension [25], salt intake [26], and possible toxic water [27], are associated with phenotypic variability (Fig. 2). Although there is wide variation in phenotypic expression and diverse factors that affect the phenotype, individual renal disease progression is associated with the kidney volume growth rate $[5,6,28,29]$.

The annual growth rate of HtTKV is estimated by eHTKV- $\alpha$, which is calculated based on a single measure- 
Fig. 2. Relation of prognostic factors that causes ESRD in ADPKD. TKV, total kidney volume; HtTKV, height-adjusted TKV; GFR, glomerular filtration rate.

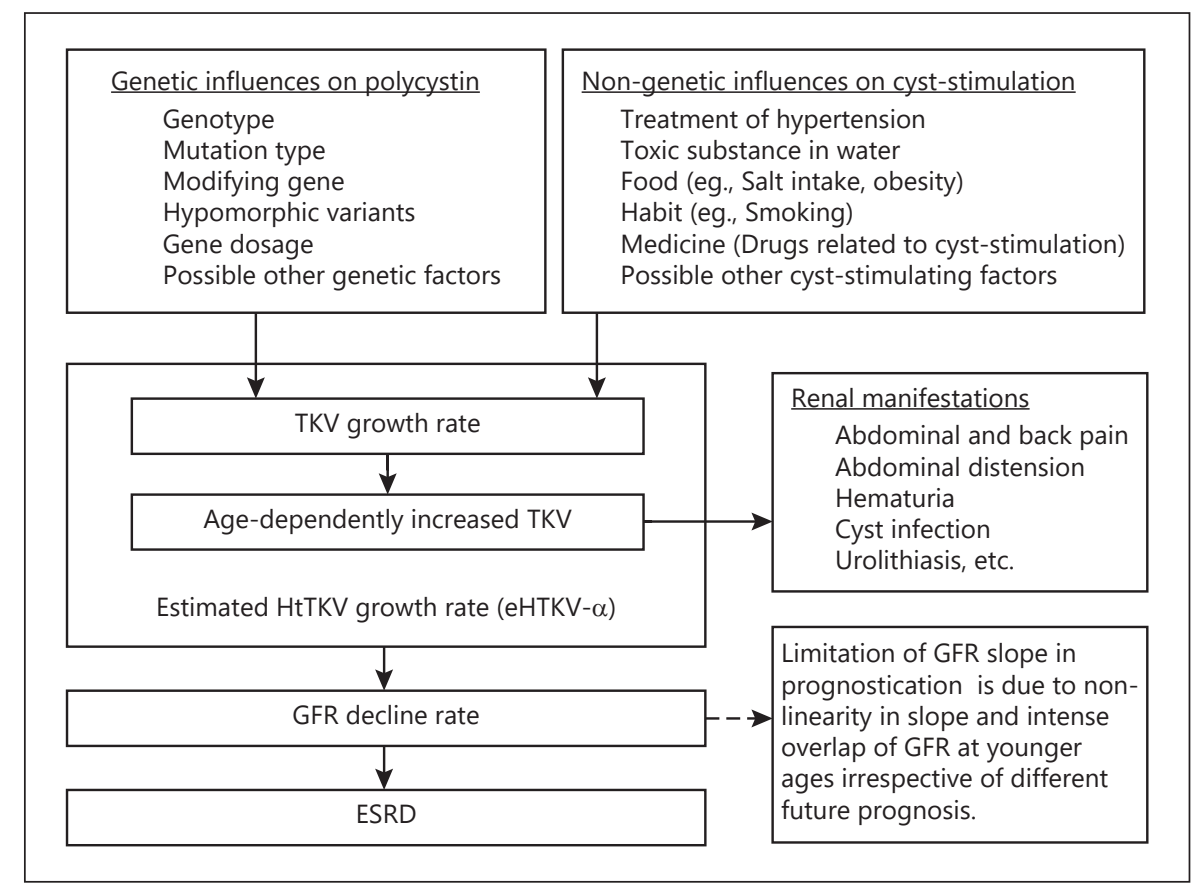

ment of kidney volume and height $[6,17]$. eHTKV- $\alpha$ is a good renal prognostic biomarker [6], which is confirmed by a high validated AUC of eHTKV- $\alpha$ for predicting ESRD in this study (Table 2; Fig. 1). The validated AUC of eHTKV- $\alpha$ is higher than that of the Predicting Renal Outcome in Polycystic Kidney Disease (PRO-PKD) score at age $65(0.84 \pm 0.02)$ [30].

Eligibility criteria can be described by a single eHTKV-a value without indexing for age and body size, since eHTKV- $\alpha$ value contains these items in itself, and without using additional risk factors such as genotype, age-dependently decreasing eGFR, eGFR slope, TKV slope, or age-dependently increasing incidence of symptoms. Patients do not need to repeat clinical tests or wait several years until judgment of eligibility.

In the ERA-EDTA guidelines [15], patients aged 4050 years with an eGFR $>60 \mathrm{~mL} / \mathrm{min} / 1.73 \mathrm{~m}^{2}$ or patients aged $30-40$ years with an eGFR $>90 \mathrm{~mL} / \mathrm{min} / 1.73 \mathrm{~m}^{2}$ are considered slow progressors. Among the remaining subjects, at least one of the following criteria is required to diagnose rapid progression: (1) a confirmed annual eGFR decline of $\geq 5 \mathrm{~mL} / \mathrm{min} / 1.73 \mathrm{~m}^{2}$ in 1 year or $\geq 2.5 \mathrm{~mL} /$ $\mathrm{min} / 1.73 \mathrm{~m}^{2}$ over a period of 5 years; (2) a TKV increase of $>5 \% /$ year by repeated measurements (preferably 3 or more); (3) Mayo Class 1C, 1D, or 1E; (4) kidney length assessed by ultrasound of $>16.5 \mathrm{~cm}$ in patients aged $<45$ years; and/or (5) having a truncating PKD1 mutation in conjunction with PRO-PKD score $>6$.
The eGFR is not appropriate predictor for individual renal prognosis even with an index for age because (1) during ages 20-40, it remains within comparable levels with slow progressors $\left(>90 \mathrm{~mL} / \mathrm{min} / 1.73 \mathrm{~m}^{2}\right)$ and starts to decline at a rate comparable with moderate to rapid progressors $[6,29]$; (2) even in subjects aged 30-40 with an eGFR $>90 \mathrm{~mL} / \mathrm{min} / 1.73 \mathrm{~m}^{2}$, ESRD developed in a substantial number [16]; (3) it declines in a nonlinear pattern in a considerable fraction [19]; and (4) the eGFR is acutely influenced by nonrenal factors such as overhydration, dehydration, and heavy exercise and by transient renal factors such as renal infection and urinary tract obstruction. These factors limit the reliability of eGFR and eGFR slope in prognostication of renal survival.

The annual TKV slope varies considerably and actual TKV growth patterns fluctuate year to year, which look like measurement errors [29]. The TKV growth rate estimated by eHTKV- $\alpha$ is more stable than the TKV growth rate estimated using $2 \mathrm{TKV}$ observations because the TKV measurement fluctuation is spread over 20 or more years in the former case but is only spread over 1 or 2 years in the latter [17, 29].

The PRO-PKD score uses genetic and clinical data. Genetic analysis is not generally used for clinical practice, and clinical manifestation is not used for subjects younger than 35 years or those missing clinical data [30]. The lower accuracy of PRO-PKD score than eHTKV-a might be due to the weak association of ADPKD genotype than 
the rate of kidney volume growth with the development of ESRD (Fig. 2) [31].

In the practical guide of the US group, Mayo Class 1C, $1 \mathrm{D}$, and $1 \mathrm{E}$ are classified as rapidly progressing disease and are recommended for tolvaptan treatment [16]. The difference between the US guidelines and this study is the inclusion of subjects with an eHTKV- $\alpha$ of 3.0-3.5\%/year $(K=150 \mathrm{~mL} / \mathrm{m})$ into rapid progressors in the US guidelines. In the Canadian guidelines, tolvaptan is recommended for Mayo Class 1C to 1E. However, additional risk factors, such as an annual decrease in eGFR of $>2.5$ $\mathrm{mL} / \mathrm{min} / 1.73 \mathrm{~m}^{2}$ and/or increase in TKV of $>5 \%$ per year, are required only for patients classified into 1C [18]. Additional conditions for $1 \mathrm{C}$ in Canadian guidelines may be unnecessary when the cutoff point of eHTKV- $\alpha$ is changed from 3.0 to $3.5 \% /$ year $(K=150 \mathrm{~mL} / \mathrm{m})$.

Approximately $93.4 \%(K=130 \mathrm{~mL} / \mathrm{m})$ or $94.6 \%(K=$ $150 \mathrm{~mL} / \mathrm{m}$ ) of the subjects likely progress to ESRD by age 65 are distinguished as the high-risk ESRD subgroup (true positive) (Table 5). High sensitivity is preferable because this cutoff point disregards the few $(5-7 \%)$ patients who progress to ESRD from the indication of tolvaptan treatment.

A relatively low specificity suggests that only $45.6-$ $47.8 \%$ (for $K=130$ and age 65 in Table 5) of subjects who do not progress to ESRD by 65 years is included to nonhigh-risk ESRD group. Rest of subjects (52.2-54.4\%) who do not progress to ESRD by age 65 is included to high-risk group (high false-positive rate). However, the eGFR decreases at a rate of more than $2.0 \mathrm{~mL} / \mathrm{min} / 1.73 \mathrm{~m}^{2}$ per year in subjects of this category (online suppl. Table 1) and ESRD may develop after 65 years. Beneficial treatment effects of tolvaptan were confirmed in the population within this eHTKV- $\alpha$ limit $[17,32]$. In addition, the subjects included in high-risk category by these cutoff points are less than half of total subjects ( $45 \%$ at $K=130$ and $49 \%$ at $K=150$ ). Inclusion of approximately half of patients for slowing the rate of renal functional decline by medical treatment seems to be permissible.

A single eHTKV-a value is advantageous for prognosticating ESRD because of the following points: (1) the accuracy of eHTKV- $\alpha$ in predicting ESRD is high. (2) It is calculated by a single measurement of TKV, and an index of age or body size is not required. (3) Limitations of using risk factor(s), such as eGFR, slope of eGFR, TKV, and TKV slope, can be avoided. (4) The measurement fluctuation of eHTKV- $\alpha$ is smaller than that of the TKV slope obtained by 2 or more measurements of TKV.

In conclusion, an initially measured eHTKV- $\alpha$ of $\geq 4.0 \% /$ year $(K=130 \mathrm{~mL} / \mathrm{m})$ or $\geq 3.5 \% /$ year $(K=150$
$\mathrm{mL} / \mathrm{m}$ ) defines high-risk ESRD. Without additional conditions, a single eHTKV- $\alpha$ cutoff point can identify adult subjects that are most likely to benefit from tolvaptan.

\section{Acknowledgements}

We thank the hundreds of patients who gave informed consent to the study and Ms. Ayako Hirao for secretary assistance. Dr. Horie was supported by Grant-in-Aid for Scientific Research (18K09151).

\section{Statement of Ethics}

This study was approved by the IRB of Kyorin University (1046-04) and Juntendo University (19-148).

\section{Conflict of Interest Statement}

Dr. Higashihara reports consulting fees from Sanofi Genzyme and grant support from Otsuka Pharmaceuticals and Taisho Pharmaceutical. Dr. Horie reports consulting fees from Sanofi, Endowment Chair, grant support from Otsuka Pharmaceuticals, and Honorarium from Chugai. Dr. Muto reports grant support from Otsuka Pharmaceuticals.

\section{Author Contributions}

Research idea and study design: E.H., S.H., and S.M.; data acquisition: S.M., H.K., M.T., T.Y., S.T., K.Y., T.Y., and H.F.; data analysis/interpretation: E.H., S.H., S.K., and T.F.; statistical analysis: T.F. and E.H.; each author contributed important intellectual content during manuscript drafting or revision, accepts personal accountability for the author's own contributions, and agrees to ensure that questions pertaining to the accuracy or integrity of any portion of the work are appropriately investigated and resolved.

$\begin{array}{ll}\text { References } \quad 1 \text { Grantham JJ. Clinical practice. Autosomal } \\ \text { dominant polycystic kidney disease. N Engl J } \\ \text { Med. 2008 Oct;359(14):1477-85. } \\ 2 \text { Chebib FT, Torres VE. Autosomal dominant } \\ \text { polycystic kidney disease: core curriculum } \\ & 2016 \text {. Am J Kidney Dis. 2016 May;67(5):792- } \\ & 810 . \\ 3 & \text { Cornec-Le Gall E, Audrézet MP, Chen JM, } \\ & \text { Hourmant M, Morin MP, Perrichot R, et al. } \\ & \text { Type of PKD1 mutation influences renal out- } \\ & \text { come in ADPKD. J Am Soc Nephrol. } \\ & \text { 2013May;24(6):1006-13. }\end{array}$


4 Spithoven EM, Kramer A, Meijer E, Orskov B, Wanner C, Caskey F, et al. Analysis of data from the ERA-EDTA Registry indicates that conventional treatments for chronic kidney disease do not reduce the need for renal replacement therapy in autosomal dominant polycystic kidney disease. Kidney Int. 2014 Dec;86(6):1244-52.

5 Grantham JJ, Torres VE, Chapman AB, Guay-Woodford LM, Bae KT, King BF Jr, et al. Volume progression in polycystic kidney disease. N Engl J Med. 2006 May;354(20): 2122-30.

6 Irazabal MV, Rangel LJ, Bergstralh EJ, Osborn SL, Harmon AJ, Sundsbak JL, et al. Imaging classification of autosomal dominant polycystic kidney disease: a simple model for selecting patients for clinical trials. J Am Soc Nephrol. 2015 Jan;26(1):160-72.

7 Grantham JJ. Rationale for early treatment of polycystic kidney disease. Pediatr Nephrol. 2015 Jul;30(7):1053-62.

8 Torres VE, Chapman AB, Devuyst O, Gansevoort RT, Grantham JJ, Higashihara E, et al. Tolvaptan in patients with autosomal dominant polycystic kidney disease. N Engl J Med. 2012 Dec;367(25):2407-18.

9 Pharmaceuticals and Medical Devices Agency [Internet]. Tokyo: Prescribing Information (in Japanese); [cited 2020 July 30]. Available from: https://www.pmda.go.jp/PmdaSearch/ iyakuDetail/ResultDataSetPDF/180078_2139 011D1022_1_08.

10 Horie S, Mochizuki T, Muto S, Hanaoka K, Fukushima Y, Narita I, et al. Erratum to: Evidence-based clinical practice guidelines for polycystic kidney disease 2014. Clin Exp Nephrol. 2016 Aug;20(4):510-09.

11 Otsuka Canada Pharmaceutical Inc [Internet]. Quebec: Product Monograph JINARC (tolvaptan); [cited 2020 July 30]. Available from: https://otsukacanada.com/wp-content/uploads/2019/09/JINARC-EN-PM.pdf.

12 European Medicines Agency [Internet]. Amsterdam: EU Summary of product characteristics Jinarc epar product information; [cited 2020 July 30]. Available from: https://www. ema.europa.eu/en/documents/product-information/jinarc-epar-product-information_en.pdf.

13 Torres VE, Chapman AB, Devuyst O, Gansevoort RT, Perrone RD, Koch G, et al. Tolvaptan in later-stage autosomal dominant polycystic kidney disease. N Engl J Med. 2017 Nov;377(20):1930-42.
14 US Food and Drug Administration [Internet]. Maryland: Highlights of prescribing information JYNARQUE; [cited 2020 July 30]. Available from: https://www.otsuka-us.com/ media/static/JYNARQUE-PI.pdf.

15 Gansevoort RT, Arici M, Benzing T, Birn H, Capasso G, Covic A, et al. Recommendations for the use of tolvaptan in autosomal dominant polycystic kidney disease: a position statement on behalf of the ERA-EDTA working groups on inherited kidney disorders and european renal best practice. Nephrol Dial Transplant. 2016 Mar;31(3):337-48.

16 Chebib FT, Perrone RD, Chapman AB, Dahl NK, Harris PC, Mrug M, et al. A practical guide for treatment of rapidly progressive ADPKD with tolvaptan. J Am Soc Nephrol. 2018 Oct;29(10):2458-70.

17 Higashihara E, Fukuhara H, Ouyang J, Lee J, Nutahara K, Tanbo M, et al. Estimation of changes in kidney volume growth rate in autosomal dominant polycystic kidney disease. Kidney Int Reports. 2020; 5: 1459-1471.

18 Soroka S, Alam A, Bevilacqua M, Girard LP, Komenda P, Loertscher R, et al. Updated Canadian expert consensus on assessing risk of disease progression and pharmacological management of autosomal dominant polycystic kidney disease. Can J Kidney Health Dis. 2018 Oct; 5:

19 Brosnahan GM, Abebe KZ, Moore CG, Rahbari-Oskoui FF, Bae KT, Grantham JJ, et al. Patterns of kidney function decline in autosomal dominant polycystic kidney disease: a post hoc analysis from the HALT-PKD trials. Am J Kidney Dis. 2018 May;71(5):666-76.

20 Higashihara E, Nutahara $K$, Okegawa T, Shishido T, Tanbo M, Kobayasi K, et al. Kidney volume and function in autosomal dominant polycystic kidney disease. Clin Exp Nephrol. 2014 Feb;18(1):157-65.

21 Matsuo S, Imai E, Horio M, Yasuda Y, Tomita K, Nitta K, et al. Revised equations for estimated GFR from serum creatinine in Japan. Am J Kidney Dis. 2009 Jun;53(6):98292.

22 Blanche P, Latouche A, Viallon V. Time-dependent AUC with right-censored data: a survey In: Lee ML, Gail M, Pfeiffer R, Satten G, Cai T, Gandy A, editors. Risk assessment and evaluation of predictions. Lecture notes in statistics. New York: Springer; 2013. Vol. 215; p. $239-51$.
23 Uno H, Cai T, Pencina MJ, D’Agostino RB, Wei LJ. On the C-statistics for evaluating overall adequacy of risk prediction procedures with censored survival data. Stat Med. 2011 May;30(10):1105-17.

24 Bergmann C, von Bothmer J, Ortiz Brüchle N, Venghaus A, Frank V, Fehrenbach H, et al. Mutations in multiple PKD genes may explain early and severe polycystic kidney disease. J Am Soc Nephrol. 2011 Nov;22(11): 2047-56.

25 Schrier RW, Abebe KZ, Perrone RD, Torres VE, Braun WE, Steinman TI, et al. Blood pressure in early autosomal dominant polycystic kidney disease. N Engl J Med. 2014 Dec; 371(24):2255-66.

26 Torres VE, Abebe KZ, Schrier RW, Perrone $\mathrm{RD}$, Chapman $\mathrm{AB}, \mathrm{Yu} A \mathrm{~A}$, et al. Dietary salt restriction is beneficial to the management of autosomal dominant polycystic kidney disease. Kidney Int. 2017 Feb;91(2):493-500

27 Gattone VH 2nd, Bacallao RL. Dichloroacetate treatment accelerates the development of pathology in rodent autosomal recessive polycystic kidney disease. Am J Physiol Renal Physiol. 2014 Nov;307(10):F1144-8.

28 Grantham JJ, Chapman AB, Torres VE. Volume progression in autosomal dominant polycystic kidney disease: the major factor determining clinical outcomes. Clin J Am Soc Nephrol. 2006 Jan;1(1):148-57.

29 Higashihara E, Yamamoto K, Kaname S, Okegawa T, Tanbo M, Yamaguchi T, et al. Age- and height-adjusted total kidney volume growth rate in autosomal dominant polycystic kidney diseases. Clin Exp Nephrol. 2019 Jan;23(1):100-11.

30 Cornec-Le GE, Audrézet MP, Rousseau A, Hourmant M, Renaudineau E, Charasse C, et al. The PROPKD score: a new algorithm to predict renal survival in autosomal dominant polycystic kidney disease. J Am Soc Nephrol. 2016 Mar;27(3):942-51.

31 Yu ASL, Shen C, Landsittel DP, Harris PC, Torres VE, Mrug M, et al. Baseline total kidney volume and the rate of kidney growth are associated with chronic kidney disease progression in Autosomal Dominant Polycystic Kidney Disease. Kidney Int. 2018 March; 93(3):691-9.

32 Irazabal MV, Blais JD, Perrone RD, Gansevoort RT, Chapman AB, Devuyst O, et al. Prognostic enrichment design in clinical trials for autosomal dominant polycystic kidney disease: The TEMPO 3:4 clinical trial. Kidney Int Rep. 2016 Aug; 1(4):213-20. 